\title{
Connective tissue growth factor gene expression and decline in renal function in lupus nephritis
}

\author{
CHIRAPORN TACHAUDOMDACH ${ }^{1}$, SURASAK KANTACHUVESIRI ${ }^{2}$, SIRIBHA CHANGSIRIKULCHAI ${ }^{3}$, \\ SURANGKANA WIMOLLUCK ${ }^{2}$, KOSET PINPRADAP $^{4}$ and CHAGRIYA KITIYAKARA ${ }^{2}$ \\ ${ }^{1}$ Molecular Medicine Graduate Program, Faculty of Science, Mahidol University; \\ ${ }^{2}$ Department of Medicine, Ramathibodi Hospital, Mahidol University; \\ ${ }^{3}$ Renal Unit, Department of Medicine, Faculty of Medicine, Srinakharinwirot University; \\ ${ }^{4}$ Department of Pathology, Faculty of Medicine, Ramathibodi Hospital, Mahidol University, Bangkok, Thailand
}

Received October 31, 2011; Accepted January 18, 2012

DOI: $10.3892 /$ etm.2012.473

\begin{abstract}
In lupus nephritis (LN), kidney inflammation may be followed by fibrosis and progressive decline in function. Transforming growth factor (TGF)- $\beta$ is a notable mediator of fibrosis, but it has other beneficial roles, thus indicating a need for alternate therapeutic targets for inhibition of fibrosis. Connective tissue growth factor (CTGF) acts as a downstream mediator of TGF- $\beta$ in promoting fibrosis, without mediating the immunosuppressive effects of TGF- $\beta$. Animal studies show that CTGF may have important roles in renal fibrosis, but data are limited in human subjects. The present study tested the hypothesis that renal CTGF mRNA expression is related to TGF- $\beta 1$ and collagen I expression and is predictive of renal function deterioration in patients with $\mathrm{LN}(\mathrm{n}=39)$. Gene expression was measured using multiplex real-time quantitative RT-PCR and renal function was estimated using the Chronic Kidney Disease Epidemiology Collaboration (CKD-EPI) glomerular filtration rate (GFR) equation. Decline in GFR was assessed by regression of GFR at biopsy to 1 year following biopsy. CTGF mRNA expression was significantly correlated with TGF- $\beta 1$ and collagen I. GFR at biopsy was $89.2 \pm 39.2 \mathrm{ml} /$ min. Renal CTGF mRNA expression correlated inversely with baseline GFR. Renal CTGF mRNA was significantly higher in patients with moderate to severe CKD compared to those in the milder CKD group (low GFR 4.92 \pm 4.34 vs. high GFR $1.52 \pm 1.94, \mathrm{p}<0.005)$. CTGF mRNA was also higher in patients

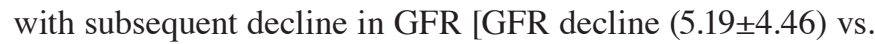
no GFR decline $(1.79 \pm 1.97) ; \mathrm{P}<0.01]$. In conclusion, renal expression of CTGF was positively related to TGF- $\beta 1$ and
\end{abstract}

Correspondence to: Dr Chagriya Kitiyakara, Renal Unit, Department of Medicine, Ramathibodi Hospital, Mahidol University, 270 Rama 6 Road, Bangkok 10400, Thailand

E-mail: kitiyakc@yahoo.com

Key words: lupus nephritis, kidney disease, systemic lupus erythematosus, gene expression, cytokines, fibrosis, kidney failure, connective tissue growth factor collagen I in patients with LN. Furthermore, high CTGF mRNA expression was associated with poor GFR at baseline and subsequent deterioration of kidney function. CTGF expression in the kidney may serve as an early marker for renal disease progression and could be evaluated as a target for therapeutic intervention to prevent renal failure in $\mathrm{LN}$.

\section{Introduction}

Systemic lupus erythematosus (SLE) is an autoimmune disease in which immune complex deposition leads to multisystem disease (1). Immune-mediated injury leads to inflammation of the kidneys and progressive renal failure. Lupus nephritis (LN) is a major cause of end-stage renal disease (ESRD) in young to middle-age females. The development of ESRD leading to dialysis therapy contributes to high morbidity and mortality and high cost of health care for patients with SLE. LN encompasses many forms of glomerulonephritis with both active inflammation and chronic scarring processes (2). Treatment of $\mathrm{LN}$ is difficult as there is heterogeneity in disease severity and outcome. High-dose immunosuppressive therapies are often required to suppress the inflammation; however, treatment must be individually tailored to achieve disease control while avoiding the risk of fatal infections and malignancies. A renal biopsy is used to evaluate the severity of injury and scarring and to serve as a guide to therapy (2). Although routine renal histopathological evaluation provides valuable prognostic information, individual patients even within the same categories may exhibit marked variability in long-term outcomes.

In chronic kidney disease (CKD) of all causes, the renal excretory function progressively declines as a consequence of nephron loss (3-6). Early in the course of CKD, renal tissue injury crosses a point of no return beyond which begins a positive feedback cycle of progressive nephron loss and replacement of normal tissue by interstitial scars. In LN, active inflammation may be followed by fibrosis and progressive decline in kidney function. By the time the patient presents to the clinician, the process of fibrosis may already have been initiated (7). Interstitial fibrosis has been associated with poor clinical outcome in numerous renal diseases. A number of cytokines mediate fibrosis by altering cell proliferation, survival, and 
by promoting the deposition of extracellular matrix (ECM) proteins such as collagens $(3,4,8,9)$. Genes involved in matrix expansion and fibrosis have been shown to correlate with renal failure in a number of experimental models $(3,5)$. Therapy aimed at inhibiting renal fibrogenesis may prevent decline in renal function. Numerous experimental studies support the role of transforming growth factor (TGF)- $\beta$ as an important mediator of collagen synthesis and fibrosis in response to renal injury (3-5). However, TGF- $\beta$ has other beneficial roles in immunosuppression, such that long-term suppression of TGF- $\beta$ may be hyper-inflammatory and pro-carcinogenic, thus highlighting a need for alternate therapeutic targets for inhibition of fibrosis.

Connective tissue growth factor (CTGF) is a cysteine-rich peptide in the $C C N$ (CTGF, cyr 61/cef 10, nov) family of growth factors $(6,9,10)$. CTGF is generated in vitro in renal cells by a variety of stimuli. In vitro and animal studies show that CTGF induces fibroblast proliferation, ECM synthesis and integrin expression and may therefore play significant roles in human renal diseases and fibrosis (11). CTGF acts as a downstream mediator of TGF- $\beta$ in promoting fibrosis and collagen deposition without mediating immune effects. Therefore, CTGF may be an attractive target for the prevention of fibrosis in human renal diseases. Previous cross-sectional studies have shown that CTGF expression is increased in human renal diseases $(6,10,11)$. However, the role of CTGF levels in renal disease progression and the relationship of CTGF with TGF- $\beta$ and collagen have yet to be evaluated in LN.

In this study, we set out to evaluate whether CTGF mRNA expression correlates with baseline renal function, and whether it is able to predict loss of renal excretory function in patients with LN. Furthermore, we examined whether renal CTGF mRNA correlates with TGF- $\beta 1$ and collagen I expression in vivo in patients with LN. In LN, the presence of established interstitial fibrosis often implies poor outcome, but when such processes are finally demonstrable on routine histology, the process is already largely irreversible (7). Findings that renal CTGF expression predicts renal outcome may pave a way for therapeutic interventions aimed at decreasing CTGF and preventing renal failure.

\section{Materials and methods}

Patient assessment and management. Patients with a formal diagnosis of SLE undergoing kidney biopsy at Ramathibodi Hospital for clinical indications between 2002 and 2004 were included in this study. An additional core of tissue samples obtained for diagnosis was snap-frozen and stored in liquid nitrogen. Control subjects with preserved renal function (serum creatinine $<1.2 \mathrm{mg} / \mathrm{dl}$ ) were recruited from patients undergoing nephrectomy for renal cell carcinoma. A tissue core was obtained from the renal cortex of the non-involved pole of the kidney, snap-frozen and stored in liquid nitrogen. This study was approved by the Ethics Committee of Ramathibodi Hospital, Faculty of Medicine. All participants gave written informed consent.

The kidney biopsy tissues were evaluated for routine histology and immunofluorescence and were classified according to the RPS/ISN criteria (12) by a specialist nephropathologist who was blinded to the clinical outcome. In addition, the activity index (AI) and chronicity index (CI) were also assessed $(12,13)$.

Patients were managed at the physicians' discretion. In general, patients received antihypertensive therapy if their blood pressure exceeded 130/80 $\mathrm{mmHg}$, and received immunosuppressive therapy according to the RPS/ISN category of glomerulonephritis. Routine clinical characteristics for each patient were recorded at baseline and at each follow-up.

\section{Kidney function assessment}

The severity of proteinuria and hematuria, which indicates inflammation of the kidneys was assessed.

Glomerular filtration rate (GFR). This value was used to estimate renal excretory function. Previous studies have identified GFR as the preferred index of renal function as it correlates most closely with clinical outcomes and other metabolic parameters (14). GFR cannot be measured directly in clinical practice. Instead, GFR was estimated using a serum creatinine-based equation adjusted for coefficients for age and gender by the Chronic Kidney Disease Epidemiology Collaboration (CKD-EPI) formula (15), which has been shown to be the most accurate GFR equation:

\section{$\mathrm{GFR}=141 \times \min (\operatorname{Scr} / \kappa, 1)^{\alpha} \times \max (\operatorname{Scr} / \kappa, 1)^{-1.209} \times 0.993^{\text {Age }} \times$ 1.018 [if female] x 1.159}

Where Scr is serum creatinine ( $\mathrm{mg} / \mathrm{dl}), \kappa$ is 0.7 for females and 0.9 for males, $\alpha$ is -0.329 for females and -0.411 for males, min indicates the minimum of $\mathrm{Scr} / \kappa$ or 1 , and max indicates the maximum of $\mathrm{Scr} / \mathrm{\kappa}$ or 1 .

Patients were also divided by severity into those with high GFR and low GFR using a cut-off eGFR of $<60 \mathrm{ml} /$ $\mathrm{min} / 1.73 \mathrm{~m}^{2}$ according to the K/DOQI guidelines (14) in which GFR $<60 \mathrm{ml} / \mathrm{min} / 1.73 \mathrm{~m}^{2}$ constitutes those with moderate to severe CKD.

Rate of GFR change ( $\triangle G F R)$. This was calculated by the slope of the GFR plot from biopsy to one year following biopsy. We divided patients into those with a decline in GFR (negative values) and those with no decline (positive values).

RNA extraction and cDNA synthesis. Total RNA was isolated from kidney tissue by silica gel-based membrane spin technology with DNase I treatment (RNeasy micro kit: Qiagen, Chatsworth, CA, USA). cDNA was synthesized with an iScripts cDNA synthesis kit using MMLV-derived reverse transcriptase pre-mixed with an RNase inhibitor (Bio-Rad, Hercules, CA, USA) using a blend of oligo (dT) and random hexamer primers. Only kidney cortex tissues with optimal quality RNA (ratio of the absorbance at 260 and $280 \mathrm{~nm}$ ranging from 1.9-2.1) were included in the study.

Quantification of renal fibrogenic gene expression. Messenger RNA expression was quantified by real-time quantitative polymerase chain reaction (RT-PCR) using a Fast Start Universal Probe Master kit (Applied Biosystems, Foster City, CA, USA). cDNA was amplified using an iScripts cDNA synthesis kit (Bio-Rad) in a 96-well plate. Multiplex quantitative PCR was performed using target and housekeeping genes in the same well (16). By this method, the threshold cycle $(\mathrm{Ct})$ of target genes in each sample was 
Table I. Primers and probe sequences.

\begin{tabular}{|c|c|c|c|}
\hline Genes & Accession no. & Primer (Forward/Reverse) & Product length (bp) \\
\hline TGF- $\beta 1$ & NM_000660.4 & $\begin{array}{l}\text { F: 5'-CCAGCATCTGCAAAGCTC } \\
\text { R: 5'-GTCAATGTACAGCTGCCGCA } \\
\text { Probe: 5'- (6-FAM)ACACCAACTATTGCTTCAGCTCCACGGA (Tamra) }\end{array}$ & 100 \\
\hline COL1 & NM_000088.3 & $\begin{array}{l}\text { F: 5'-CCTCAA GGCTCCAACGAG } \\
\text { R: 5'-TCAATCACTGTCTTGCCCCA } \\
\text { Probe: 5'- (6-FAM)ATGGCTGCACGAGTCACACCGGA (Tamra) }\end{array}$ & 117 \\
\hline CTGF & NM_001901.2 & $\begin{array}{l}\text { Assay-by-design (Applied Biosystems: ABI) } \\
\text { Assay ID: Hs00170014m1 } \\
\text { Probe: 5'-(6-FAM)AGAACATTAAGAAGGGCAAAAAGTG (Tamra) }\end{array}$ & 60 \\
\hline
\end{tabular}

normalized with the housekeeping gene to account for individual sample variation in the RNA amount. Ct was defined as the cycle number at which the fluorescence intensity generated by the tracer dye, which is released from the probe during DNA amplification, reaches a fixed limit of detection. $\mathrm{Ct}$ was determined at the exponential phase and is inversely related to the initial mRNA amount.

Primer and probe sequences for CTGF, TGF- $\beta 1$ and collagen I are shown in Table I. The mRNA expression of the target genes was calculated by using the $\Delta \mathrm{Ct}$ procedure with VIC-TAMRA-labeled GAPDH (Applied Biosystems) used as a housekeeping gene for normalization among samples where $\Delta \mathrm{Ct}$ values were calculated by the formula:

$$
\Delta \mathrm{Ct}=\mathrm{Ct}_{\text {target gene }}-\mathrm{Ct}_{\text {housekeeping gene }}
$$

A standard curve was generated for each gene using pooled cDNA. Conditions of the PCR reaction were optimized so that the amplification efficiency of target genes and the endogenous reference genes were comparable across $3 \log$ dilutions of pooled cDNA for each pair of target and housekeeping genes (17). The optimized conditions were as follows: TGF- $\beta 1$ or COL1, $250 \mathrm{nM}$ primers and $250 \mathrm{nM}$ probes, with $1 \mu 1$ GAPDH. The PCR conditions were: $50^{\circ} \mathrm{C}$ for $2 \mathrm{~min}, 95^{\circ} \mathrm{C}$ for $10 \mathrm{~min}$, followed by 40 cycles at $95^{\circ} \mathrm{C}$ for $0.15 \mathrm{~min}$ and $60^{\circ} \mathrm{C}$ for $1 \mathrm{~min}$.

The mean $\mathrm{Ct}$ of triplicates was used. The relative levels of gene expression in $\mathrm{LN}$ patients were evaluated using the comparative cycle $(\mathrm{Ct})$ method, also known as the $2^{-\Delta \Delta \mathrm{CT}}$ method (17). The expression of target genes in LN tissues were expressed as fold change of the mean value of the expression of control subjects ( $n=3$ ), which were arbitrarily set as 1 as follows: Relative expression to control tissues $=2^{-\Delta \Delta \mathrm{CT}}$, where:

$$
\begin{aligned}
\Delta \mathrm{Ct} & =\mathrm{Ct}_{\text {target gene }}-\mathrm{Ct}_{\text {housekeeping gene }} \\
\Delta \Delta \mathrm{Ct} & =\Delta \mathrm{Ct}_{\mathrm{LN} \text { tissues }}-\Delta \mathrm{Ct}_{\text {control tissues }}
\end{aligned}
$$

Outcomes. The levels of CTGF gene expression were correlated with baseline GFR and other clinical parameters, and with $\triangle$ GFR. In order to examine the potential mechanisms for CTGF actions, the levels of CTGF expression were also correlated with collagen I and TGF- $\beta 1$.

Statistical analysis. Data are summarized as the means \pm $\mathrm{SD}$, and as percentages. Correlations were calculated using Spearman's rank coefficient. Student's t-test or nonparametric tests were used to compare differences between groups for continuous data. Statistical significance was defined to be indicated by $\mathrm{p}<0.05$, two-tailed. Statistical analysis was conducted using the software package SPSS version 15.0 (SPSS, Inc., Chicago, IL, USA).

\section{Results}

Patient characteristics. Patient characteristics are summarized in Table II. Of the 39 patients included in the study, the majority were women $(n=38)$. Their age at the time of biopsy was $31.4 \pm 10.6$ years (Table II). Time of disease onset to kidney biopsy was $4.6 \pm 4.9$ years. The majority (87\%) had received prior treatment with either prednisolone or immunosuppressive agents. Systolic BP was $130.3 \pm 13.5 \mathrm{mmHg}$. Diastolic $\mathrm{BP}$ was $80.5 \pm 10.2 \mathrm{mmHg}$. Hypertension was observed in $53.9 \%$ of patients (BP $>140 / 90)$ and the serum creatinine level was $1.7 \pm 2.3 \mathrm{mg} / \mathrm{dl}$. GFR at the time of biopsy was $89.2 \pm 39.2 \mathrm{ml} / \mathrm{min}$. Of the 39 patients, $8(20.5 \%)$ had low GFR $\left(\right.$ GFR $\left.<60 \mathrm{ml} / \mathrm{min} / 1.73 \mathrm{~m}^{2}\right)$ and $31(79.5 \%)$ had high GFR $\left(\mathrm{GFR} \geq 60 \mathrm{ml} / \mathrm{min} / 1.73 \mathrm{~m}^{2}\right.$ ). Proteinuria was $2.5 \pm 4.1 \mathrm{~g} / 24 \mathrm{~h}$. Urine RBC was $2.7 \pm 2.3 / \mathrm{HPF}$.

One patient had mixed V+II classes and was designated as class II. There were 7 (17.9\%) patients with class II, 3 (7.7\%) with class III, 18 (46.2\%) with class IV, 9 (23.1\%) with class V and $2(5.1 \%)$ with class VI LN. Activity index (AI) was 4.2 \pm 5.3 ; chronicity index $(\mathrm{CI})$ was $2.0 \pm 1.7$.

The majority of patients received oral or intravenous cyclophosphamide $(n=27)$. Other treatment protocols were: azathioprine + corticosteroids $(n=5)$, mycophenolate mofetil + corticosteroids $(n=2)$ and corticosteroid alone $(n=5)$. GFR at the 1-year follow-up was $81.3 \pm 38.0 \mathrm{ml} / \mathrm{min} / 1.73 \mathrm{~m}^{2}$.

CTGF gene expression and correlations with TGF- $\beta 1$ and collagen I. No PCR products were formed when reactions were performed without reverse transcriptase, indicating that the primers and probes did not amplify genomic DNA. The slope of the linear regression of the graph for $\Delta \mathrm{Ct}$ against the log concentrations of pooled cDNA was $<0.1$, indicating that the amplification efficiencies of target and housekeeping genes were comparable (data not shown). The $2^{-\Delta \Delta C T}$ method can therefore be used to assess comparative gene expression (17). Mean \%CV for the $\mathrm{Ct}$ were $0.01-0.02 \%$.

Renal CTGF expression tended to be higher in LN than the controls ( $\mathrm{LN} 4.21 \pm 4.17$ vs. controls $1.49 \pm 1.17$-fold, $\mathrm{p}=0.072$ ). 
Table II. Baseline patient characteristics $(n=39)$.

Age at initial biopsy (years)
Time from disease onset to biopsy
Female patients $(\%)$
Prior immunosuppressive agent
Systolic BP (mmHg)
Diastolic BP $(\mathrm{mmHg})$
Hypertensive $(\mathrm{BP}>140 / 90)(\%)$
Serum albumin $(\mathrm{g} / \mathrm{l})$
Creatinine $(\mathrm{mg} / \mathrm{dl})$
eGFR (ml/min/1.73 $\left.\mathrm{m}^{2}\right)$
eGFR <60 $\mathrm{ml} / \mathrm{min} / 1.73 \mathrm{~m}^{2}(\%)$
Urine protein $(\mathrm{g} / \mathrm{day})$
Kidney biopsy
RPS/ISN class $\mathrm{n}(\%)$
II
III
IV
V
VI

Activity index

Chronicity index

Outcome

GFR at 1 year

Rate of change of GFR
A

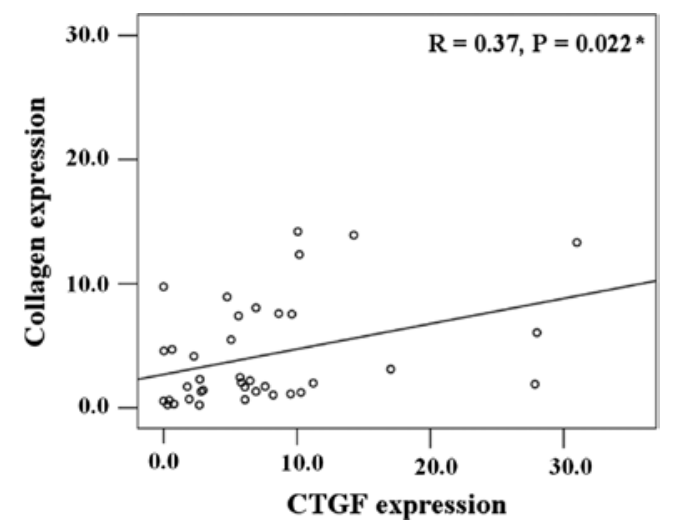

B

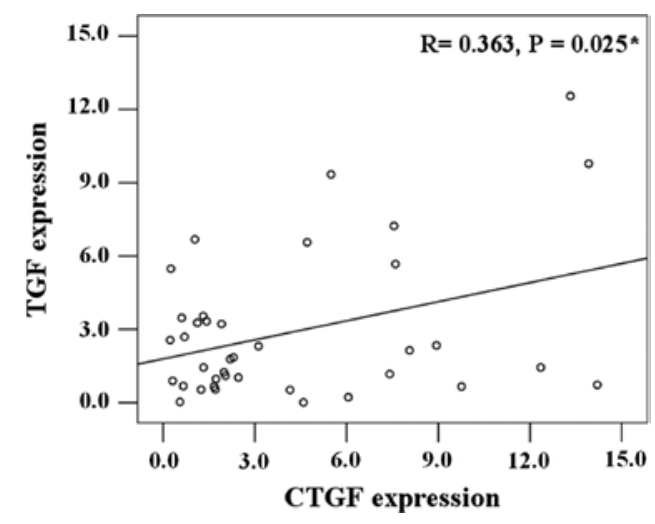

Figure 1. Correlations between renal CTGF mRNA expression and other fibrogenic genes in lupus nephritis $(n=39)$. mRNA expression of (A) collagen $I$ and (B) transforming growth factor (TGF)- $\beta$.
In LN subjects, the mRNA expression of CTGF and COL1 in $\mathrm{LN}$ tissues were positively correlated $(\mathrm{R}=0.370, \mathrm{p}=0.022)$ (Fig. 1A). In addition, the mRNA expression of CTGF and TGF- $\beta 1$ in LN tissues were positively correlated $(\mathrm{R}=0.363$, $\mathrm{p}=0.025)$ (Fig. 1B).

Renal CTGF mRNA expression and baseline clinicopathological parameters. There were no significant correlations between CTGF and the degree of proteinuria or hematuria. There were no significant differences in CTGF expression between classes and no correlations with $\mathrm{AI}$ or $\mathrm{CI}$.

CTGF expression correlated inversely with GFR at baseline $(\mathrm{R}=-0.41, \mathrm{p}=0.009)$ (Fig. 2). When patients were divided into severity groups, CTGF expression was significantly higher in patients with moderate to severe CKD compared to those with milder forms of CKD (low GFR 4.92 \pm 4.34 vs. high GFR $1.52 \pm 1.94, \mathrm{p}=0.005)$ (Fig. 3).

Renal CTGF mRNA expression and rate of decline in GFR. In patients with LN, the rate of change in GFR was $-7.94 \pm 31.13 \mathrm{ml} / \mathrm{min} / 1.73 \mathrm{~m}^{2}$ per year. Overall, 29 cases exhibited a decline in GFR. CTGF expression was significantly higher in patients with a decline in GFR (decline 5.19 \pm 4.46 vs. no decline 1.79 $\pm 1.97, \mathrm{p}=0.010$ ) (Fig. 4).

\section{Discussion}

CTGF has been implicated in matrix expansion and fibrosis in a number of experimental models $(6,9,10)$, but data are limited in patients. The major novel findings of this study were that renal CTGF expression was increased in LN, that the level of CTGF expression correlated inversely with renal excretory function at the time of biopsy, and that the gene expression levels were higher in those with subsequent decline in kidney function.

Numerous experimental studies support the role of TGF- $\beta 1$ as an important mediator of fibrosis (3-5). Upregulated expression of TGF- $\beta 1$ is a feature of virtually all human and experimental models of renal fibrosis including murine lupus models (18). Early studies revealed that TGF- $\beta 1$ increased CTGF mRNA levels markedly in human foreskin fibroblasts (19) and subsequent reports identified TGF- $\beta$-responsive elements in the promoter region of the CTGF gene (6,9-11). CTGF is likely to act in part as a downstream mediator of TGF- $\beta 1$ fibrogenic activity, including the induction of fibroblast proliferation, transcription of gene-encoding matrix genes, and inhibition of matrix-degrading enzymes. Previous studies have shown that the addition of recombinant CTGF to mesangial cells increased the expression of ECM components including collagen I (20). The latter is normally present in relatively small amounts in adult kidneys (21). Accumulation of collagen I has been reported in the kidneys of patients with native kidney diseases (22) and those with chronic allograft nephropathy (23). An important role for CTGF in renal fibrosis is supported by the observation that transgenic mice with overexpression of CTGF develop renal fibrosis (24). Furthermore, inhibition of CTGF by antisense oligodeoxynucleotides effectively reduced expression of ECM genes, and tubulointerstitial fibrosis in experimental renal diseases (25). The relationship 


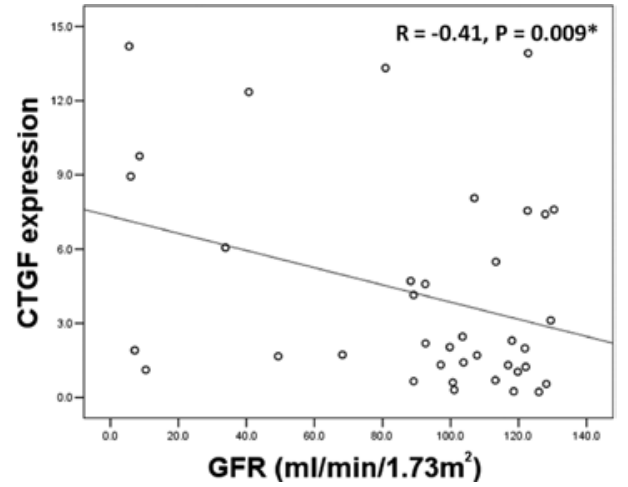

Figure 2. Correlations between renal CTGF mRNA expression and glomerular filtration rate in lupus nephritis $(n=39)$.

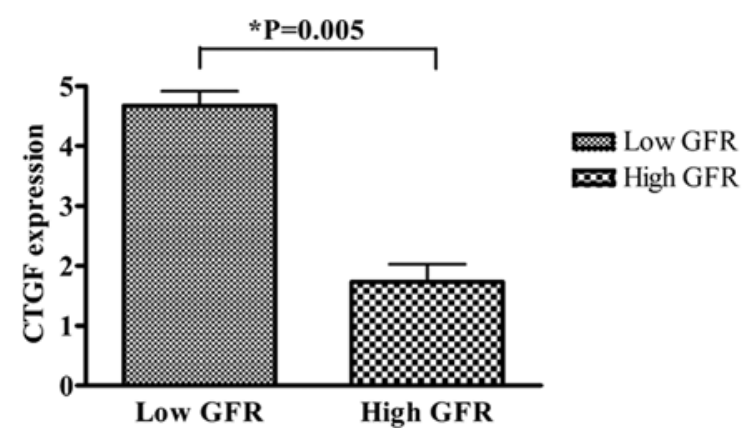

Figure 3. Renal CTGF mRNA expression in patients with mild chronic kidney disease (GFR $\geq 60 \mathrm{ml} / \mathrm{min} / 1.73 \mathrm{~m}^{2}$ ) vs. moderate or severe chronic kidney disease (GFR $<60 \mathrm{ml} / \mathrm{min} / 1.73 \mathrm{~m}^{2}$ ).

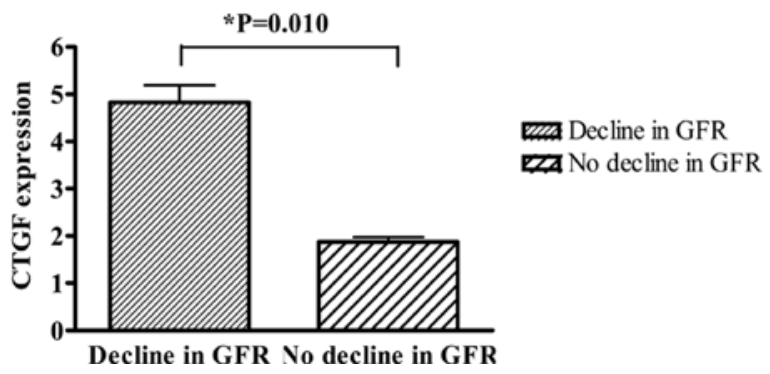

Figure 4. Renal CTGF mRNA expression in patients with a decline in GFR and with no decline in GFR.

between TGF- $\beta$, collagens and CTGF has previously not been extensively studied in human glomerulonephritis. The present study revealed that the levels of expression of collagen I and TGF- $\beta 1$ are correlated with CTGF, supporting the theory that, as with experimental animals, these fibrogenic pathways are interrelated in human LN.

In normal human kidneys, CTGF mRNA is weakly expressed in podocytes, in certain parietal epithelial cells, and in interstitial cells (26). Upregulation of CTGF was observed in numerous experimental models $(6,9,10)$. In the murine lupus nephritis model, renal CTGF and collagen expression were increased (18). Both genes were suppressed by complement inhibition consistent with a link between immune activation and subsequent fibrosis. Limited data are available on the association between CTGF expression in human kidney diseases, and the above-mentioned studies did not examine the relationship between gene expression and renal function or disease progression. Suzuki et al documented increases in renal CTGF mRNA expression in patients with diabetic nephropathy and IgA nephropathy, but not in minimal change disease (27). Kobayashi et al reported that CTGF expression was increased in renal biopsies of patients with type 2 diabetic nephropathy, and that the increased expression correlated with baseline serum creatinine and interstitial fibrosis (28). Ito et al examined renal expression of CTGF mRNA by in situ hybridization in a variety of biopsies of patients with chronic glomerulopathies and tubulointerstitial damage. They found that strong overexpression of CTGF correlated with the degree of scarring (26). Only six subjects with LN were included in the latter study and no data on renal function were available for these subjects. Therefore, the present study extended previous clinical and animal studies by showing that CTGF expression correlated inversely with GFR in human subjects with LN. Furthermore, the increase in CTGF predicted those patients likely to undergo a decline in GFR after 1-year follow-up. This may provide a useful marker of poor disease outcome and allow clinicians to use a more aggressive treatment or extend more specific anti-fibrotic therapy to such patients. This is particularly important, as $\mathrm{LN}$ is a heterogeneous disease with regard to responses to treatment and long-term outcome. Previous studies have revealed that, although routine histology of the kidney provides beneficial prognostic information, the predictive values of various clinicopathological indices were not universally consistent in different studies (2).

TGF- $\beta 1$ is the driving force behind renal fibrosis and is regarded as a key target in prevention and treatment of renal fibrosis. However, in addition to its fibrogenic role as the main inducer of ECM production, the functions of TGF- $\beta 1$ also include regulation of cell growth, cell differentiation, cytokine production, and immune cell modulation (8-10). Considering its multiple effects, which are in part also protective and beneficial, direct inhibition of general TGF- $\beta$ activity might not be clinically useful. Thus, therapeutic strategies that are aimed at targeting the downstream effectors of TGF- $\beta$ that specifically mediate its fibrogenic action, such as CTGF, appear to be promising targets for modulation of profibrotic TGF- $\beta$ activity. Recently, a first phase I trial was carried out to treat patients with diabetic kidney disease using a human monoclonal antibody to CTGF. The human monoclonal antibody was shown to be well tolerated and associated with short-term beneficial effects (29). The findings of the present study support the possibility that inhibition of CTGF may also be considered in LN.

In conclusion, CTGF expression in LN correlated with TGF- $\beta 1$ and collagen I and inversely correlated with renal function. CTGF expression in the kidney may serve as an early marker for GFR decline in LN. If the findings of this study are confirmed, patients with high CTGF may be subjected to more aggressive therapy with standard immunosuppression to prevent fibrosis. Alternatively, studies aimed at directly inhibiting CTGF could be considered as a strategy to prevent renal failure in $\mathrm{LN}$ patients.

\section{Acknowledgements}

C.T. was supported by grants from the Strategic Scholarships for Frontier Research Network for the Joint PhD Program 
Thai Doctoral degree, and the Office of the Higher Education Commission. C.K. was supported by grants from the Office of the Higher Education Commission, and Thailand Research Fund (RMU4880048), Mahidol University, and Ramathibodi Hospital. S.K. was supported by the Research Fund of 'Genetic studies in transgenic animal models of SLE' (no. 02011854-0005) Mahidol University. This study was taken from a dissertation submitted by Chiraporn Tachaudomdach to fulfill requirements for the $\mathrm{PhD}$ degree from Mahidol University in 2011.

\section{References}

1. D'Cruz DP, Khamashta MA and Hughes GR: Systemic lupus erythematosus. Lancet 17: 587-596, 2007.

2. Markowitz GS and D'Agati VD: Classification of lupus nephritis. Curr Opin Nephrol Hypertens 18: 220-225, 2009.

3. Boor P, Ostendorf T and Floege J: Renal fibrosis: novel insights into mechanisms and therapeutic targets. Nat Rev Nephrol 6: 643-656, 2010.

4. Garcia-Sanchez O, Lopez-Hernandez FJ and Lopez-Novoa JM: An integrative view on the role of TGF-beta in the progressive tubular deletion associated with chronic kidney disease. Kidney Int 77: 950-955, 2010.

5. Zeisberg M and Neilson EG: Mechanisms of tubulointerstitial fibrosis. J Am Soc Nephrol 21: 1819-1834, 2010.

6. Goldschmeding R, Aten J, Ito Y, Blom I, Rabelink $T$ and Weening JJ: Connective tissue growth factor: just another factor in renal fibrosis? Nephrol Dial Transplant 15: 296-299, 2000.

7. Hill GS, Delahousse M, Nochy D, et al: Outcome of relapse in lupus nephritis: roles of reversal of renal fibrosis and response of inflammation to therapy. Kidney Int 61: 2176-2186, 2002.

8. Michael Z, Gary BYM, Pablo C, et al: Collagen and fibrosis. J Urol 67: 176-184, 2005.

9. Phanish MK, Winn SK and Dockrell ME: Connective tissue growth factor-(CTGF, CCN2) - a marker, mediator and therapeutic target for renal fibrosis. Nephron Exp Nephrol 114: 83-92, 2010.

10. Chen XM, Qi W and Pollock CA: CTGF and chronic kidney fibrosis. Front Biosci 1: 132-141, 2009.

11. Gupta S, Clarkson MR, Duggan J and Brady HR: Connective tissue growth factor: potential role in glomerulosclerosis and tubulointerstitial fibrosis. Kidney Int 58: 1389-1399, 2000.

12. Weening JJ, D'Agati VD, Schwartz MM, et al: The classification of glomerulonephritis in systemic lupus erythematosus revisited. J Am Soc Nephrol 15: 241-250, 2004.

13. Austin HA, Muenz LR, Joyce KM, Antonovych TT and Balow JE: Diffuse proliferative lupus nephritis: identification of specific pathologic features affecting renal outcome. Kidney Int 25: 689-695, 1984.

14. National Kidney Foundation: K/DOQI clinical practice guidelines for chronic kidney disease: evaluation, classification, and stratification. Am J Kidney Dis 39: S1-266, 2002.
15. Levey AS, Stevens LA, Schmid CH, et al: A new equation to estimate glomerular filtration rate. Ann Intern Med 5: 604-612, 2009.

16. Li YL, Ye F, Hu Y, Lu WG and Xie X: Identification of suitable reference genes for gene expression studies of human serous ovarian cancer by real-time polymerase chain reaction. Anal Biochem 394: 110-116, 2009.

17. Livak KJ and Schmittgen TD: Analysis of relative gene expression data using real-time quantitative PCR and the 2(-Delta Delta C(T)) Method. Methods 25: 402-408, 2001.

18. Bao L, Zhou J, Holers VM and Quigg RJ: Excessive matrix accumulation in the kidneys of MRL/lpr lupus mice is dependent on complement activation. J Am Soc Nephrol 14: 2516-2525, 2003.

19. Igarashi A, Okochi H, Bradham DM and Grotendorst GR: Regulation of connective tissue growth factor gene expression in human skin fibroblasts and during wound repair. Mol Biol Cell 4: 637-645, 1993.

20. Murphy M, Godson C, Cannon S, et al: Suppression subtractive hybridization identifies high glucose levels as a stimulus for expression of connective tissue growth factor and other genes in human mesangial cells. J Biol Chem 274: 5830-5834, 1999.

21. Furness PN: Extracellular matrix and the kidney. J Clin Pathol 49: 355-359, 1996.

22. Vleming LJ, Baelde JJ, Westendorp RG, Daha MR, van Es LA and Bruijn JA: Progression of chronic renal disease in humans is associated with the deposition of basement membrane components and decorin in the interstitial extracellular matrix. Clin Nephrol 44: 211-219, 1995.

23. Bakker RC, Koop K, Sijpkens YW, et al: Early interstitial accumulation of collagen type I discriminates chronic rejection from chronic cyclosporine nephrotoxicity. J Am Soc Nephrol 14: 2142-2149, 2003

24. Sonnylal S, Shi-Wen X, Leoni P, et al: Selective expression of connective tissue growth factor in fibroblasts in vivo promotes systemic tissue fibrosis. Arthritis Rheum 62: 1523-1532, 2010.

25. Yokoi H, Mukoyama M, Nagae T, et al: Reduction in connective tissue growth factor by antisense treatment ameliorates renal tubulointerstitial fibrosis. J Am Soc Nephrol 15: 1430-1440, 2004.

26. Ito Y, Aten J, Bende RJ, et al: Expression of connective tissue growth factor in human renal fibrosis. Kidney Int 53: 853-861, 1998.

27. Suzuki D, Toyoda M, Umezono T, et al: Glomerular expression of connective tissue growth factor mRNA in various renal diseases. Nephrology 8: 92-97, 2003.

28. Kobayashi T, Okada H, Inoue T, Kanno Y and Suzuki H: Tubular expression of connective tissue growth factor correlates with interstitial fibrosis in type 2 diabetic nephropathy. Nephrol Dial Transplant 21: 548-549, 2006.

29. Adler SG, Schwartz S, Williams ME, et al: Phase 1 study of anti-CTGF monoclonal antibody in patients with diabetes and microalbuminuria. Clin J Am Soc Nephrol 5: 1420-1428, 2010. 